were the presenting manifestations in $54(87 \%) ; 7$ had a history of headache, and 5 had been treated for epilepsy. (Hladky JP et al. Child's Nerv Syst 1994;10:328-333; Ped Neur Briefs Sept 1994). AVMs were supratentorial in $41(79 \%)$ and infratentorial in $11(21 \%)$. The smaller the AVM, the higher the risk of hemorrhage, and the greater the need for early diagnosis and surgical resection. Postoperative angiography is recommended at 1 year after surgery to exclude recurrence, and after 5 years for diffuse AVMs.

\title{
SPONTANEOUS INTRACRANIAL ARTERIAL DISSECTION
}

A 14 year-old male with intracranial carotid artery dissection had transient neurologic symptoms and no antecedent illness or trauma, as reported from the University of Kentucky. Lexington. Diagnosis made by dynamic CT was confirmed by catheter arteriography. The dissection involved the supraclinoid segment of the left internal carotid. Vasculitis, prothrombotic states, and collagen defects were excluded as possible causes. (Robertson WC Jr, Given CA II. Spontaneous intracranial arterial dissection in the young: diagnosis by CT

angiography. BMC Neurol 2006;6:16). (Respond: William C Robertson Jr: wcrobe2@email.uky.edu).

COMMENT. Occlusion of the supraclinoid segment of the internal carotid artery is a common arteriographic finding in children with acute hemiplegia. Associated disorders include pre-existing heart disease, trauma, CNS infection, sickle cell disease, and moyamoya disease, but a high proportion are idiopathic.

Traumatic vertebral artery dissection may present with vomiting, occipital headache, stiff neck, and ataxia. A review of 19 published cases found 1 died, 2 had residual quadriplegia, 9 had mild to moderate hemiparesis, ataxia, and/or dysarthria, and 7 (37\%) recovered (Garg BP et al. Strokes in children due to vertebral artery trauma. Neurology 1993;43:2555-2558; Ped Neur Briefs Jan 1994).

\section{TEMPORAL LOBE EPILEPSY}

\section{FACTORS PREDICTIVE OF SEIZURE OUTCOME IN NEW-ONSET TEMPORAL LOBE EPILEPSY}

A community-based cohort of 77 children with new-onset temporal lobe epilepsy (TLE) were followed prospectively and reviewed at 7 and 14 years after seizure onset, and clinical, EEG, and neuroimaging findings and seizure outcome are reported from the Royal Children's Hospital and University of Melbourne, Australia, and Starship Children's Hospital, Auckland, New Zealand. Age at follow-up was a median of 20 years (range, 12 to 29 years), and the median follow-up period was 13.7 years. Of 62 patients completing the study, $19(30 \%)$ were seizure free and off treatment, with no seizures for 5 to 15 years, while 43 had ongoing seizures or were treated surgically. MRI lesions identified in 28 patients with seizures included hippocampal sclerosis in 10 , tumor in 8 , and cortical dysplasia in 7 . Focal slowing on the EEG was also associated with persistent seizures. Twenty one $(75 \%)$ patients with positive neuroimaging studies underwent surgery, and $67 \%$ became seizure-free. Factors 